\title{
Positive solutions for nonlinear fractional differential equation with nonlocal boundary conditions
}

\author{
Noureddine Bouteraa ${ }^{a^{*}}$, Slimane Benaicha ${ }^{\mathrm{a}}$ and Habib Djourdem ${ }^{\mathrm{a}}$ \\ ${ }^{a}$ Laboratory of Fundamental and Applied Mathematics of Oran (LMFAO), University of Oran1, Ahmed Benbella, Algeria \\ *Corresponding author E-mail: bouteraa-27@hotmail.fr
}

\author{
Article Info \\ Keywords: Positive Solution, Iterative \\ sequence, Green's function \\ 2010 AMS: Primary 34B18, Secondary \\ $34 A 08,26 A 33$ \\ Received: 18 February 2018 \\ Accepted: 1 March 2018 \\ Available online: 11 March 2018
}

\begin{abstract}
In this paper, we study the boundary value problem of a class of fractional differential equations involving the Riemann-Liouville fractional derivative with nonlocal integral boundary conditions. To establish the existence results for the given problems, we use the properties of the Green's function and the monotone iteration technique, one shows the existence of positive solutions and constructs two successively iterative sequences to approximate the solutions. The results are illustrated with an example.
\end{abstract}

\section{Introduction}

In this paper, we are interested in the existence of solutions for the nonlinear fractional differential equation

$$
D_{0^{+}}^{\alpha} u(t)+f(t, u(t))=0, \quad t \in(0,1),
$$

subject to the boundary conditions

$$
u^{(i)}(0)=0,0 \leq i \leq 2, D_{0^{+}}^{\beta} u(1)=\lambda I_{0^{+}}^{\beta} u(\eta),
$$

where $D_{0^{+}}^{\alpha}, D_{0^{+}}^{\beta}$ are the standard Riemann-Liouville fractional derivative of order $\alpha \in(3,4], \beta \in(0,2], I_{0+}^{\beta}$ is the standard Riemann-Liouville fractional integral of order $\beta \in(0,2]$ and $0 \leq \frac{\lambda \Gamma(\alpha-\beta) \eta^{\alpha+\beta-1}}{\Gamma(\alpha+\beta)}<1$.

The first definition of fractional derivative was introduced at the end of the nineteenth century by Liouville and Riemann, but the concept of non-integer derivative and integral, as a generalization of the traditional integer order differential and integral calculus, was mentioned already in 1695 by Leibniz and L'Hospital. In fact, fractional derivatives provide an excellent tool for the description of memory and hereditary properties of various materials and processes. The mathematical modelling of systems and processes in the fields of physics, chemistry, aerodynamics, electrodynamics of complex medium, polymer rheology, Bode's analysis of feedback amplifiers, capacitor theory, electrical circuits, electro-analytical chemistry, biology, control theory, fitting of experimental data, involves derivatives (or q-derivatives) of fractional order see for example [5, 6]. For more details we refer the reader to $[2,11]$ and the references cited therein.

Many mathematicians show strong interest in fractional differential equations and many wonderful results have been obtained. The techniques of nonlinear analysis, as the main method to deal with the problems of nonlinear fractional differential equations, plays an essential role in the research of this field, such as establishing the existence and the uniqueness or the multiplicity of solutions to nonlinear fractional differential equations boundary value problems, see $[4,7,9,10,11,12,15,16,17,18,19]$ and the references therein.

In [3], the authors studied the boundary value problems of the fractional order differential equation:

$$
\left\{\begin{array}{c}
D_{0^{+}}^{\alpha} u(t)+f(t, u(t))=0, \quad t \in(0,1), \\
u(0)=0, D_{0^{+}}^{\beta} u(1)=a D_{0^{+}}^{\beta} u(\eta),
\end{array}\right.
$$


where $1<\alpha \leq 2,0<\eta<1,0<a, \beta<1, f \in C\left([0,1] \times \mathbb{R}^{2}, \mathbb{R}\right)$ and $D_{0^{+}}^{\alpha}, D_{0^{+}}^{\beta}$ are the standard Riemann-Liouville fractional derivative of order $\alpha, \beta$ respectively. They obtained the multiple positive solutions by the Leray-Schauder nonlinear alternative and the fixed point theorem on cones.

The monotone iteration scheme is an interesting and effective way to investigate the existence of solutions to nonlinear fractional problem (see for example $[8,13,14])$. Inspired and motivated by the works mentioned above, we focus on the existence of positive solutions for the nonlocal boundary value problem $(1.1)-(1.2)$ by using the fixed point theorem for increasing operators on the order intervals, we also establish two iterative sequences to approximate the solutions. The paper is organized as follows. In Section 2, we recall some preliminary facts that we need in the sequel, for more details; see [1]. The existence of the positive solutions to the problem (1.1) - (1.2), is proved and two successively iterative sequences to approximate the solutions are constructed and we give an example to illustrate our results in Section 3 .

\section{Preliminaries}

In this section, we recall some definitions and facts which will be used in the later analysis.

Definition 2.1. Let $E$ be a real Banach space. A nonempty closed set $K \subset E$ is said to be a cone provided that

(i) $c_{1} u+c_{2} v \in K$ for all $c_{1} \geq 0, c_{2} \geq 0$, and

(ii) $u \in K,-u \in K$ implies $u=0$.

Every cone $K$ induces an ordering in $E$ given by $u \leq v$ if and only if $v-u \in K$.

Definition 2.2. The Riemann-Liouville fractional integral of order $\alpha>0$ of a function $u:(0, \infty) \rightarrow \mathbb{R}$ is given by

$$
I_{0^{+}}^{\alpha} u(t)=\frac{1}{\Gamma(\alpha)} \int_{0}^{t}(t-s)^{\alpha-1} u(s) d s, \quad t>0,
$$

where $\Gamma(\cdot)$ is the Euler Gamma function, provided that the right side is pointwise defined on $(0, \infty)$.

Definition 2.3. [1]. The Riemann-Liouville fractional derivative order $\alpha>0$ of a continuous function $u:(0, \infty) \rightarrow \mathbb{R}$ is defined by

$$
D_{0^{+}}^{\alpha} u(t)=\frac{1}{\Gamma(n-\alpha)} \frac{d^{n}}{d t^{n}} \int_{0}^{t}(t-s)^{n-\alpha-1} u(s) d s, \quad t>0,
$$

where $n=\lceil\alpha\rceil+1,\lceil\alpha\rceil$ denotes the integer part of number $\alpha$, provided that the right side is pointwise defined on $(0, \infty)$.

Lemma 2.4. [1] (i) If $u \in L^{p}(0,1), 1 \leq p \leq+\infty, \beta>\alpha>0$, then $D_{0^{+}}^{\alpha} I_{0^{+}}^{\beta} u(t)=I_{0^{+}}^{\beta-\alpha} u(t), D_{0^{+}}^{\alpha} I_{0^{+}}^{\alpha} u(t)=u(t), I_{0^{+}}^{\alpha} I_{0^{+}}^{\beta} u(t)=I_{0^{+}}^{\alpha+\beta} u(t)$. (ii) If $\beta>\alpha>0$, then $D^{\alpha} t^{\beta-1}=\frac{\Gamma(\beta) t^{\beta-\alpha-1}}{\Gamma(\beta-\alpha)}$.

(iii) If $\alpha>0$ and $\gamma \in(-1,+\infty)$, then $I_{0^{+}}^{\alpha} t^{\gamma}=\frac{\Gamma(\gamma+1)}{\Gamma(\alpha+\gamma+1)} t^{\alpha+\gamma}$.

Lemma 2.5. [1] Let $\alpha>0$ and for any $y \in L^{1}(0,1)$. Then, the general solution of the fractional differential equation $D_{0^{+}}^{\alpha} u(t)+y(t)=$ $0,0<t<1$ is given by

$$
u(t)=-\frac{1}{\Gamma(\alpha)} \int_{0}^{t}(t-s)^{\alpha-1} y(s) d s+c_{1} t^{\alpha-1}+c_{2} t^{\alpha-2}+\cdots+c_{n} t^{\alpha-n},
$$

where $c_{0}, c_{1}, \ldots, c_{n-1}$ are real constants and $n=\lceil\alpha\rceil+1$.

Lemma 2.6. Let $y \in C[0,1]$. Then the solution of the fractional boundary value problem

$$
\left\{\begin{array}{c}
D_{0^{+}}^{\alpha} u(t)+y(t)=0, \\
u^{(i)}(0)=0,0 \leq i \leq 2, \\
D_{0^{+}}^{\beta} u(1)=\lambda I_{0^{+}}^{\beta} u(\eta),
\end{array}\right.
$$

is given by

$$
u(t)=\int_{0}^{1} G(t, s) y(s) d s
$$

where

$$
G(t, s)=\left\{\begin{array}{cl}
\frac{-P \Gamma(\alpha-\beta) \Gamma(\alpha+\beta)(t-s)^{\alpha-1}+\Delta}{P \Gamma(\alpha) \Gamma(\alpha-\beta) \Gamma(\alpha+\beta)}, & 0 \leq s \leq t \leq 1, s \leq \eta, \\
\frac{\Delta}{P \Gamma(\alpha) \Gamma(\alpha-\beta) \Gamma(\alpha+\beta)}, & 0 \leq t \leq s \leq \eta \leq 1, \\
\frac{-P \Gamma(\alpha-\beta) \Gamma(\alpha+\beta)(t-s)^{\alpha-1}+\Lambda}{P \Gamma(\alpha) \Gamma(\alpha-\beta) \Gamma(\alpha+\beta)}, & 0 \leq \eta \leq s \leq t \leq 1, \\
\frac{\Gamma(\alpha) \Gamma(\alpha+\beta)(1-s)^{\alpha-\beta-1} t^{\alpha-1}}{P \Gamma(\alpha) \Gamma(\alpha-\beta) \Gamma(\alpha+\beta)}, & 0 \leq t \leq s \leq 1, s \geq \eta,
\end{array}\right.
$$

where $\Delta=t^{\alpha-1}\left[\Gamma(\alpha) \Gamma(\alpha+\beta)(1-s)^{\alpha-\beta-1}-\lambda \Gamma(\alpha) \Gamma(\alpha-\beta)(\eta-s)^{\alpha+\beta-1}\right]$, $\Lambda=\Gamma(\alpha+\beta) \Gamma(\alpha)(1-s)^{\alpha-\beta-1} t^{\alpha-1}$.

and $P=\frac{\Gamma(\alpha)}{\Gamma(\alpha-\beta)}-\frac{\lambda \Gamma(\alpha)}{\Gamma(\alpha+\beta)} \eta^{\alpha+\beta-1}$. 
Proof. In view of Lemma 2.5, the general solution for the above equation is

$$
u(t)=-\frac{1}{\Gamma(\alpha)} \int_{0}^{t}(t-s)^{\alpha-1} y(s) d s+c_{1} t^{\alpha-1}+c_{2} t^{\alpha-2}+c_{3} t^{\alpha-3}+C_{4} t^{\alpha-4},
$$

where $c_{1}, c_{2}, c_{3}, c_{4} \in \mathbb{R}$.

The boundary conditions $u(0)=u^{\prime}(0)=u^{\prime \prime}(0)=0$, implies that $c_{2}=c_{3}=c_{4}=0$. Thus

$$
u(t)=-\frac{1}{\Gamma(\alpha)} \int_{0}^{t}(t-s)^{\alpha-1} y(s) d s+c_{1} t^{\alpha-1} .
$$

By (2.4) and Lemma 2.4, we get

$$
D_{0^{+}}^{\beta} u(t)=\frac{1}{\Gamma(\alpha-\beta)}\left[c_{1} \Gamma(\alpha) t^{\alpha-\beta-1}-\int_{0}^{t}(t-s)^{\alpha-\beta-1} y(s) d s\right] .
$$

In view of boundary condition $D_{0^{+}}^{\beta} u(1)=\lambda I_{0^{+}}^{\beta} u(\eta)$, we conclude that

$$
c_{1}=\frac{1}{P}\left[\frac{1}{\Gamma(\alpha-\beta)} \int_{0}^{1}(1-s)^{\alpha-\beta-1} y(s) d s-\frac{\lambda}{\Gamma(\alpha+\beta)} \int_{0}^{\eta}(\eta-s)^{\alpha+\beta-1} y(s) d s\right] .
$$

Therefore, the unique solution of the problem (2.1) is given by

$$
\begin{aligned}
& u(t)=\frac{t^{\alpha-1}}{P \Gamma(\alpha-\beta)} \int_{0}^{1}(1-s)^{\alpha-\beta-1} y(s) d s-\frac{\lambda t^{\alpha-1}}{P \Gamma(\alpha+\beta)} \int_{0}^{\eta}(\eta-s)^{\alpha+\beta-1} y(s) d s \\
& -\frac{1}{\Gamma(\alpha)} \int_{0}^{t}(t-s)^{\alpha-1} y(s) d s .
\end{aligned}
$$

For $t \leq \eta$, one has

$$
\begin{aligned}
& u(t)=\frac{t^{\alpha-1}}{P \Gamma(\alpha-\beta)}\left[\int_{0}^{t}(1-s)^{\alpha-\beta-1} y(s) d s+\int_{t}^{\eta}(1-s)^{\alpha-\beta-1} y(s) d s+\int_{\eta}^{1}(1-s)^{\alpha-\beta-1} y(s) d s\right] \\
& -\frac{\lambda t^{\alpha-1}}{P \Gamma(\alpha+\beta)}\left[\int_{0}^{t}(\eta-s)^{\alpha+\beta-1} y(s) d s+\int_{t}^{\eta}(\eta-s)^{\alpha+\beta-1} y(s) d s\right]-\frac{1}{\Gamma(\alpha)} \int_{0}^{t}(t-s)^{\alpha-1} y(s) d s, \\
& \quad=\int_{0}^{t} \frac{-P \Gamma(\alpha-\beta) \Gamma(\alpha+\beta)(t-s)^{\alpha-1}+\Delta}{P \Gamma(\alpha) \Gamma(\alpha+\beta) \Gamma(\alpha-\beta)} y(s) d s+\int_{t}^{\eta} \frac{\Delta}{P \Gamma(\alpha) \Gamma(\alpha+\beta) \Gamma(\alpha-\beta)} y(s) d s \\
& +\int_{\eta}^{1} \frac{\Gamma(\alpha) \Gamma(\alpha+\beta)(1-s)^{\alpha-\beta-1} t^{\alpha-1}}{P \Gamma(\alpha) \Gamma(\alpha+\beta) \Gamma(\alpha-\beta)} y(s) d s, \\
& =\int_{0}^{1} G(t, s) y(s) d s .
\end{aligned}
$$

For $t \geq \eta$, one has

$$
\begin{aligned}
& u(t)=\int_{0}^{\eta} \frac{-P \Gamma(\alpha-\beta) \Gamma(\alpha+\beta)(t-s)^{\alpha-1}+\Delta}{P \Gamma(\alpha) \Gamma(\alpha+1) \Gamma(\alpha-\beta)} y(s) d s \\
& +\int_{\eta}^{t} \frac{-P \Gamma(\alpha-\beta) \Gamma(\alpha+\beta)(t-s)^{\alpha-1}+\Gamma(\alpha) \Gamma(\alpha+\beta)(1-s)^{\alpha-\beta-1} t^{\alpha-1}}{P \Gamma(\alpha) \Gamma(\alpha+\beta) \Gamma(\alpha-\beta)} y(s) d s \\
& +\int_{t}^{1} \frac{\Gamma(\alpha) \Gamma(\alpha+\beta)(1-s)^{\alpha-\beta-1} t^{\alpha-1}}{P \Gamma(\alpha) \Gamma(\alpha+\beta) \Gamma(\alpha-\beta)} y(s) d s, \\
& =\int_{0}^{1} G(t, s) y(s) d s .
\end{aligned}
$$

The proof is complete. 
We need some properties of function $G(t, s)$ to establish the existence of positive solutions.

Lemma 2.7. The Green's function $G(t, s)$ has the following properties:

(i) The function $G(t, s)$ is continuous and $G(t, s)>0$ for all $t, s \in(0,1)$.

(ii) For all $t, s \in(0,1)$, we have

$$
t^{\alpha-1} w_{2}(s) \leq G(t, s) \leq t^{\alpha-1} w_{1}(s)
$$

where

$$
w_{1}(s)=\frac{\lambda \eta^{\alpha+\beta-1}\left[(1-s)^{\alpha-\beta-1}-(1-s)^{\alpha+\beta-1}\right]}{P \Gamma(\alpha+\beta)},
$$

and

$$
w_{2}(s)=\frac{(1-s)^{\alpha-\beta-1}}{P \Gamma(\alpha-\beta)} .
$$

Proof. It is easy to prove $(i)$. Now, we prove (ii), assume that $0 \leq \frac{\lambda \Gamma(\alpha-\beta) \eta^{\alpha+\beta-1}}{\Gamma(\alpha+\beta)}<1$, then for $0 \leq s \leq t \leq 1, s \leq \eta$, we get

$$
\begin{aligned}
P \Gamma(\alpha) \Gamma(\alpha+\beta) \Gamma(\alpha-\beta) G(t, s)= & -P \Gamma(\alpha-\beta) \Gamma(\alpha+\beta)(t-s)^{\alpha-\beta-1} \\
& +\Gamma(\alpha) \Gamma(\alpha+\beta)(1-s)^{\alpha-\beta-1} t^{\alpha-1}-\lambda \Gamma(\alpha) \Gamma(\alpha-\beta)(\eta-s)^{\alpha+\beta-1} t^{\alpha-1}, \\
= & \lambda \Gamma(\alpha) \Gamma(\alpha-\beta)\left\{\eta^{\alpha+\beta-1}(t-s)^{\alpha-1}-(\eta-s)^{\alpha+\beta-1} t^{\alpha-1}\right\} \\
& +\Gamma(\alpha) \Gamma(\alpha+\beta)\left\{-(t-s)^{\alpha-1}+(1-s)^{\alpha-\beta-1} t^{\alpha-1}\right\}, \\
\geq & \lambda \eta^{\alpha+\beta-1} \Gamma(\alpha) \Gamma(\alpha-\beta) t^{\alpha-1}\left[(1-s)^{\alpha-\beta-1}-(1-s)^{\alpha+\beta-1}\right],
\end{aligned}
$$

and

$$
\begin{aligned}
P \Gamma(\alpha) \Gamma(\alpha+\beta) \Gamma(\alpha-\beta) G(t, s) & =\Gamma(\alpha) \Gamma(\alpha+\beta)(1-s)^{\alpha-\beta-1} t^{\alpha-1}-\lambda \Gamma(\alpha) \Gamma(\alpha-\beta)(\eta-s)^{\alpha+\beta-1} t^{\alpha-1} \\
& \leq \Gamma(\alpha) \Gamma(\alpha+\beta)(1-s)^{\alpha-\beta-1} t^{\alpha-1} .
\end{aligned}
$$

For $0 \leq \eta \leq s \leq t \leq 1$, we have

$$
\begin{aligned}
P \Gamma(\alpha) \Gamma(\alpha+\beta) \Gamma(\alpha-\beta) G(t, s) & =-P \Gamma(\alpha-\beta) \Gamma(\alpha+\beta)(t-s)^{\alpha-1}+\Gamma(\alpha) \Gamma(\alpha+\beta)(1-s)^{\alpha-\beta-1} t^{\alpha-1} \\
& =-\Gamma(\alpha) \Gamma(\alpha+\beta)(t-s)^{\alpha-1}+\lambda \Gamma(\alpha) \Gamma(\alpha-\beta) \eta^{\alpha+\beta-1}(t-s)^{\alpha-1} \\
& +\Gamma(\alpha) \Gamma(\alpha+\beta)(1-s)^{\alpha-\beta-1} t^{\alpha-1} \\
& \geq \lambda \Gamma(\alpha) \Gamma(\alpha-\beta) \eta^{\alpha+\beta-1}(t-s)^{\alpha-1}-\Gamma(\alpha) \Gamma(\alpha-\beta) \eta^{\alpha+\beta-1}(t-s)^{\alpha-1} \\
& +\Gamma(\alpha) \Gamma(\alpha-\beta) \eta^{\alpha+\beta-1}(1-s)^{\alpha-\beta-1} t^{\alpha-1} \\
& \geq \lambda \Gamma(\alpha) \Gamma(\alpha-\beta) \eta^{\alpha-\beta-1}(1-s)^{\alpha-\beta-1} t^{\alpha-1} \\
& \geq \lambda \Gamma(\alpha) \Gamma(\alpha-\beta) \eta^{\alpha+\beta-1} t^{\alpha-1}\left[(1-s)^{\alpha-\beta-1}-(1-s)^{\alpha+\beta-1}\right],
\end{aligned}
$$

and

$$
\begin{aligned}
P \Gamma(\alpha) \Gamma(\alpha+\beta) \Gamma(\alpha-\beta) G(t, s) & =-P \Gamma(\alpha-\beta) \Gamma(\alpha+\beta)(t-s)^{\alpha-1}+\Gamma(\alpha) \Gamma(\alpha+\beta)(1-s)^{\alpha-\beta-1} t^{\alpha-1}, \\
& =-\Gamma(\alpha) \Gamma(\alpha+\beta)(t-s)^{\alpha-1} t^{\alpha-1}+\lambda \Gamma(\alpha) \Gamma(\alpha-\beta) \eta^{\alpha+\beta-1}(t-s)^{\alpha-1} \\
& +\Gamma(\alpha) \Gamma(\alpha+\beta)(1-s)^{\alpha-\beta-1} t^{\alpha-1}, \\
& \leq \Gamma(\alpha) \Gamma(\alpha+\beta)(1-s)^{\alpha-\beta-1} t^{\alpha-1} .
\end{aligned}
$$

For $0 \leq t \leq s \leq 1, s \geq \eta$, we get

$$
\begin{aligned}
P \Gamma(\alpha) \Gamma(\alpha+\beta) \Gamma(\alpha-\beta) G(t, s) & =\Gamma(\alpha) \Gamma(\alpha+\beta)(1-s)^{\alpha-\beta-1} t^{\alpha-1} \\
& \geq \lambda \Gamma(\alpha) \Gamma(\alpha-\beta) \eta^{\alpha+\beta-1} t^{\alpha-1}\left[(1-s)^{\alpha-\beta-1}-(1-s)^{\alpha+\beta-1}\right]
\end{aligned}
$$




\section{Existence results}

We shall consider the Banach space $E=C[0,1]$ equipped with the norm $\|u\|=\max _{0 \leq t \leq 1}|u(t)|$ and let a closed cone $K \subset E$ by $K=$ $\{u \in E: u \geq 0\}$ where 0 is the the zero function. Then $K$ is normal.

Set $K_{a}=\{u \in E:\|u\| \leq a\}$. Define the operator $T: K_{a} \rightarrow E$ as

$$
(T u)(t)=\int_{0}^{1} G(t, s) f(s, u(s)) d s, \quad t \in[0,1],
$$

where $G(t, s)$ is given by (2.3). It is not hard to see that fixed points of operator $T$ coincide with the solutions to the problem (1.1) - (1.2).

Lemma 3.1. [9] Let $E$ be a Banach space ordered by a normal cone $K \subset$ E. Assume that $T:\left[x_{1}, x_{2}\right] \rightarrow E$ is completely continuous operator such that $x_{1} \leq T x_{2}, x_{2} \geq T x_{2}$. Then $T$ has a minimal fixed point $x_{*}$ and a maximal fixed point $x^{*}$ such that $x_{1} \leq x_{*} \leq x^{*} \leq x_{2}$. Moreover, $x_{*}=\lim _{n \rightarrow \infty} T^{n} x_{1}, \quad x^{*}=T^{n} x_{2}$, where $\left\{T^{n} x_{1}\right\}_{n=1}^{\infty}$ is an increasing sequence and $\left\{T^{n} x_{2}\right\}_{n=1}^{\infty}$ is a decreasing sequence.

First, for the existence results of problem $(1.1)-(1.2)$, we need the following assumptions.

$\left(A_{1}\right) f:[0,1] \times[0, a] \rightarrow[0, \infty)$ is continuous and $f(t, 0) \neq 0$,

$\left(A_{2}\right)$ There exists a nonnegative function $q \in C[0,1] \subseteq L^{1}[0,1]$ such that $|f(t, u)| \leq q(t),(t, u) \in[0,1] \times[0, a]$,

$\left(A_{3}\right) f(t, \underline{u}) \leq f(t, \bar{u}), t \in[0,1], 0 \leq \underline{u} \leq \bar{u} \leq a$.

Lemma 3.2. Assume that $\left(A_{1}\right)-\left(A_{3}\right)$ hold. Then the operator $T$ defined in $(3.1)$ is a completely continuous increasing operator.

Proof. Firstly, the operator $T$ is continuous in view of the continuity of functions $f(t, u(t))$ and $G(t, s)$. Secondly, we will show that $T\left(K_{a}\right)$ is bounded. Let

$$
L=\int_{0}^{1} q(t) d t<\infty
$$

Then, for any $u \in K_{a}$, we have

$$
\|(T u)(t)\|=\max _{t \in[0,1]} \int_{0}^{1} G(t, s)|f(s, u(s))| d s \leq \frac{L}{P \Gamma(\alpha-\beta)}, \quad t \in[0,1] .
$$

For each $u \in K_{a}$, one can show that

$$
\begin{aligned}
& \left|(T u)^{\prime}(t)\right|=\mid \frac{(\alpha-1) t^{\alpha-2}}{P \Gamma(\alpha-\beta)} \int_{0}^{1}(1-s)^{\alpha-\beta-1} f(s, u(s)) d s \\
& -\frac{\lambda(\alpha-1) t^{\alpha-2}}{P \Gamma(\alpha+\beta)} \int_{0}^{\eta}(\eta-s)^{\alpha+\beta-1} f(s, u(s)) d s-\frac{\alpha-1}{\Gamma(\alpha)} \int_{0}^{t}(t-s)^{\alpha-2} f(s, u(s)) d s \mid, \\
& =\mid \frac{(\alpha-1) t^{\alpha-2}}{P \Gamma(\alpha-\beta)} \int_{0}^{1}(1-s)^{\alpha+\beta-1} f(s, u(s)) d s \\
& -\frac{\lambda(\alpha-1) t^{\alpha-2}}{P \Gamma(\alpha+\beta)} \int_{0}^{\eta}(\eta-s)^{\alpha+\beta-1} f(s, u(s)) d s-\frac{1}{\Gamma(\alpha-1)} \int_{0}^{t}(t-s)^{\alpha-2} f(s, u(s)) d s \mid, \\
& \leq \frac{(\alpha-1) t^{\alpha-2}}{P \Gamma(\alpha-\beta)} \int_{0}^{1}(1-s)^{\alpha+\beta-1}|f(s, u(s))| d s \\
& +\frac{\lambda(\alpha-1) t^{\alpha-2}}{P \Gamma(\alpha+\beta)} \int_{0}^{\eta}(\eta-s)^{\alpha+\beta-1}|f(s, u(s))| d s+\frac{1}{\Gamma(\alpha-1)} \int_{0}^{t}(t-s)^{\alpha-2}|f(s, u(s))| d s, \\
& \leq \frac{(\alpha-1) L}{P \Gamma(\alpha-\beta)}+\frac{\lambda(\alpha-1) L}{P \Gamma(\alpha+\beta)}+\frac{L}{\Gamma(\alpha-1)}=\bar{L} .
\end{aligned}
$$

Therefore, for any $t_{1}, t_{2} \in\left[t_{1}, t_{2}\right] \leq \bar{L}\left(t_{2}-t_{1}\right)$. with $t_{1}<t_{2}$, we have

$$
\left|(T u)\left(t_{1}\right)-(T u)\left(t_{2}\right)\right| \leq \int_{t_{1}}^{t_{2}}\left|(T u)^{\prime}(s)\right| d s \leq \bar{L}\left(t_{2}-t_{1}\right) \rightarrow 0 \text { as } t_{2} \rightarrow t_{1},
$$

The Arzela-Ascoli theorem implies that the operator $T: K_{a} \rightarrow E$ is completely continuous. The assumption $\left(A_{3}\right)$ provides that the operator $T: K_{a} \rightarrow E$ is an increasing operator. The proof is completed. 
Theorem 3.3. Assume that $\left(A_{1}\right)-\left(A_{3}\right)$ hold, and

$$
\int_{0}^{1} w_{1}(s) f(s, 0) d s \geq 0, \quad \int_{0}^{1} w_{2}(s) f\left(a, a s^{\alpha-1}\right) \leq a, \quad s \in[0,1] .
$$

Then the problem (1.1) - (1.2) has two positive solutions $u^{*}, v^{*}$ satisfying $0<u^{*} \leq v^{*} \leq$ a. Moreover, there exist a non-decreasing iterative sequence $\left\{u_{n}\right\}_{n=0}^{\infty}$ with

$$
\lim _{n \rightarrow \infty} u_{n}=u^{*}, \quad u_{0}=0, \quad u_{n+1}=T u_{n}, \quad n=0,1,2, \ldots,
$$

and a non-decreasing iterative sequence $\left\{v_{n}\right\}_{n=0}^{\infty}$ with

$$
\lim _{n \rightarrow \infty} v_{n}=u^{*}, \quad v_{0}=a t^{\alpha-1}, \quad v_{n+1}=T v_{n}, \quad n=0,1,2, \ldots
$$

Proof. We only need to prove that $T u_{0} \geq u_{0}$ and $T v_{0} \leq u_{0}$.

$$
\begin{aligned}
\left(T u_{0}\right)(t) & =\int_{0}^{1} G(t, s) f\left(s, u_{0}\right) d s=\int_{0}^{1} G(t, s) f(s, 0) d s, \\
& \geq t^{\alpha-1} \int_{0}^{1} w_{1}(s) f(s, 0) d s \geq 0=u_{0}, \quad t \in[0,1],
\end{aligned}
$$

this implies $u_{1} \geq u_{0}$, wich combined with $\left(A_{3}\right)$ gives

$$
u_{2}=\left(T u_{1}\right)(t)=\int_{0}^{1} G(t, s) f\left(s, u_{1}(t)\right) d s \geq u_{1}, \quad t \in[0,1]
$$

Similarly, we have

$$
\begin{aligned}
v_{1} & =T v_{0}=\int_{0}^{1} G(t, s) f\left(s, v_{0}\right) d s, \\
& \leq t^{\alpha-1} \int_{0}^{1} w_{2}(s) f\left(s, a t^{\alpha-1}\right) d s, \\
& \leq a t^{\alpha-1}=v_{0}, \quad t \in[0,1]
\end{aligned}
$$

Then, by (3.5) - (3.6) and induction, the iterative sequences $\left\{u_{n}\right\},\left\{v_{n}\right\}$ satisfy

$$
u_{0}(t) \leq u_{1}(t) \leq \ldots \leq u_{n}(t) \leq \ldots \leq v_{n}(t) \leq \ldots \leq v_{1}(t) \leq v_{0}(t), \forall t \in[0,1] .
$$

By induction, one can prove that $u_{n+1} \geq u_{n}$ and $v_{n+1} \leq v_{n}$.

Lemma 3.1 shows that the operator $T$ has a minimal fixed point $u^{*}$ and a maximal fixed point $v^{*}$ satisfying $0 \leq u^{*} \leq v^{*} \leq a$. From $\left(A_{1}\right)$ we find that the zero function is not the solution to the problem $(1.1)-(1.2)$. Thus $0<u^{*} \leq v^{*} \leq a$. The proof is complete.

We construct an example to illustrate the applicability of the results presented.

Example 3.4. Consider the following boundary value problem

$$
\begin{aligned}
& D_{0^{+}}^{\frac{7}{2}} u(t)+\frac{1}{\sqrt{t}}\left(t+u^{\frac{1}{3}}(t) \tanh (u(t))+u^{\frac{1}{3}}(t)\right)=0, t \in(0,1), \\
& u(0)=u^{\prime}(0)=u^{\prime \prime}(0)=0, D^{\frac{5}{2}} u(1)=\frac{1}{2} I_{0^{+}}^{\frac{5}{2}} u\left(\frac{1}{2}\right),
\end{aligned}
$$

where $\alpha=\frac{7}{2}, \beta=\frac{5}{2}, \lambda=\frac{1}{2}, \eta=\frac{1}{2}$ and $f(t, u(t))=\frac{1}{\sqrt{t}}\left(t+u^{\frac{1}{3}}(t) \tanh (u(t))+u^{\frac{1}{3}}(t)\right)$.

We take $a=10$. By simple calculation we have

$P=3,3229182, \quad f(t, 0)=\frac{t}{\sqrt{t}}$ and $f\left(t, 10 t^{\frac{5}{2}}\right)=\frac{1}{\sqrt{t}}\left(t+(10)^{\frac{1}{3}} t^{\frac{5}{6}}+t^{\frac{5}{6}}\right)$.

A simple calculation leads to

$$
\int_{0}^{1} w_{1}(s) f(s, 0) d s \simeq 0,0000239 \geq 0,
$$

and

$$
\int_{0}^{1} w_{2}(s) f\left(s, 10 s^{\frac{5}{2}}\right) d s \simeq 0,9124781 \leq 10 .
$$

Hence, all the assumptions of Theorem 3.3 are satisfied. Which implies that the boundary value (1.1) - (2.1) has two nontrivial solutions $u^{*}, v^{*}$ with $0 \leq u^{*} \leq v^{*} \leq 10$, and the two monotone iterative sequences $\left\{u_{n}\right\}_{n=1}^{\infty}$ can be taken as

$$
u_{0}=0, \quad u_{n+1}=T u_{n}, \quad v_{0}=10 t^{\alpha-1}, \quad v_{n+1}=T v_{n}, \quad n=0,1,2, \ldots
$$




\section{Acknowledgments}

The authors want to thank the anonymous referee for the throughout reading of the manuscript and several suggestions that help us improve the presentation of the paper.

\section{References}

[1] A. A. Kilbas, H. M. Srivastava, J. J. Trijullo, Theory and applications of fractional differential equations, Elsevier Science b. V, Amsterdam, (2006). 1.

[2] A. Cabada, G. Wang, positive solutions of nonlinear fractional differential equations with integral boundary conditions. J. Math. Anal. Appl. 389(1), (2012), 403-411.

[3] C. F. Li, X. N. Luo and Y. Zhou, Existence of positive solutions of the boundary value problem for nonlinear fractional differential equations. Comput. Math. Appl. 59 (2010), 1363-1375.

[4] F. Liu, K. Burrage, Novel techniques in parameter estimation for fractional dynamycal models arising from biological systems Comput. Math. Appl. 62(3), (2011), 822-833.

[5] J. R. Graef, L. Kong, Q. Kong and M. Wang, Uniqueness of positive solutions of fractional boundary value problems with non-homogeneous integral boundary conditions, Fract. Calc. Appl. Anal. 15 (2012), 509-528.

[6] J. R. Graef, L. Kong, Q. Kong, Positive solution for a class of higher-order boundary value problems with fractional q-derivatives. Appl. Math. Comput. 218 (2012), 9682-9689.

[7] J. R. Graef, L. Kong, Q. Kong, and M. Wang, Existence and uniqueness of solutions for a fractional boundary value problem with Dirichlet boundary condition, Electron. J. Qual. Theory Differ. Equ. 2013 No.55,11 pp.

[8] M. Al-Refai, M. Hadjji, Monotone iterative sequences for nonlinear boundary value problems of fractional order. Nonlinear Anal, 74 (2011), $3531-3539$.

[9] J. Tan and C. Cheng, Fractional boundary value problems with Riemann-Liouville fractional derivatives. Bound. Value Probl. Doi: 13662-015-0413-y, (2015), 14 pages.

[10] N. Bouteraa and S. Benaicha, Existence of solutions for three-point boundary value problem for nonlinear fractional differential equations, An. Univ. Oradea, fasc. Math. Volume 25 (2018), nr. 1. to appear.

[11] S. Miller, B. Ross, An introduction to the fractional calculus and fractional differential equations, John Wiley and Sons, Inc.; New York, (1993). 1, 1.3, 1

[12] S. G. Samko, A. A. Kilbas and O. I. Marichev, Fractional Integrals and Derivatives: Theory and Applications. Gordon \& Breach, Yverdon (1993).

[13] S. liu, H. Li and Q. Dai, Nonlinear fractional differential equations with nonlocal integral boundary conditions, Bound.Value. Prob. (2015), 11 pages.

[14] V. Lakshmikantham and A. S. Vatsala, General uniqueness and monotone iterative technique for fractional differential equations. Appl. Math. Lett. 21 (8) (2008), 828-834.

[15] X. L. Han, H. L. Gao, Existence of positive solutions for eigenvalue problem of nonlinear fractional differential equations. Adv. Differ. Equ. 201266 (2012).

[16] Y. Sun, M. Zhao, Positive solutions for a class of fractional differential equations with integral boundary conditions. Appl. Math. Lett, 34 (2014), 17-21.

[17] Y. F. Xu, Fractional boundary value problems with integral and anti-periodic boundary conditions. Bull. Malays. Math. Sci. Soc. 39, (2016), 571-587.

[18] Y. Qiao and Z. zhou, Existence of solutions for a class of fractional differential equations with integral and anti-periodic boundary conditions, Bound. Value Probl. Doi: 13661-016-0547-x, (2017), 9 pages.

[19] Z. B. Bai, W. C. Sun and W. Zhang, Positive solutions for boundary value problems of singular fractional differential equations. Abstr. Appl. Anal. 2013, Article ID 129640 (2013). 